\title{
Role of Vitamins and Minerals in Improving Immunity during Covid-19 Pandemic - A Review
}

\author{
Archana Dhok ${ }^{1}$, Lata Kanyal Butolaㄹ, Ashish Anjankar 3 , Amol Datta Rao Shinde4, \\ Prakash Kesharao Kute ${ }^{5}$, Roshan Kumar Jha ${ }^{6}$
}

\begin{abstract}
1Department of Biochemistry, Jawaharlal Nehru Medical College, Datta Meghe Institute of Medical Sciences, Sawangi, Wardha, Maharashtra, India. ${ }^{2}$ Department of Biochemistry, Jawaharlal Nehru Medical College,

Datta Meghe Institute of Medical Sciences, Sawangi, Wardha, Maharashtra, India. ${ }^{3}$ Department of Biochemistry, Jawaharlal Nehru Medical College, Datta Meghe Institute of Medical Sciences, Sawangi, Wardha, Maharashtra, India. ${ }^{4}$ Department of Biochemistry, Jawaharlal Nehru Medical College, Datta Meghe Institute of Medical Sciences, Sawangi, Wardha, Maharashtra, India. ${ }^{5}$ Department of Biochemistry, Jawaharlal Nehru Medical College, Datta Meghe Institute of Medical Sciences, Sawangi, Wardha, Maharashtra, India. ${ }^{6}$ Department of Biochemistry, Jawaharlal Nehru Medical College, Datta Meghe Institute of Medical Sciences, Sawangi, Wardha, Maharashtra, India.
\end{abstract}

ABSTRACT

\section{BACKGROUND}

The COVID-19 pandemic has brought attention to the role of the immune system. The immune system can be strengthened by diet, supplements and good hygiene practices. The immune system is comprised of different cells contained in the blood, lymph, and tissues that are distributed across the body. A large number of cells and even greater numbers of chemical messengers engage in the body's immune defence. In immune response blood cells, B-lymphocytes and T- lymphocytes play a vital role. The world health organization, urges people to follow a healthier lifestyle, saying it will increase their chances of a quick recovery. Proper nutrition is required for proper functioning of immune system. Therefore, healthy balanced diet is the best way to support the immune function. Vitamins and minerals are important ingredients of the diet that the body requires to work properly. Vit D is required in limited amounts to maintain good health. Vitamins and minerals together are also called micronutrients, as they are required in very small quantities. On the other hand, carbs, proteins and fats are altogether termed as macronutrients. In recent years, vitamins A, C, D, and E, believed to have an effect on the immune system, have gained significant attention because they are involved in enhancement of immune systems. Solely, vit-A is involved in proper functioning of eye, immune system and also takes care of skin and genes. In green leafy vegetables, carrots, pumpkin, melons and mangoes vitamins are present in form of pre-vitamins, and further converted into active vit A inside the human body. It is surprising that liver, yolk of egg, butter, whole milk, and cheese are active source of vitamin A. Having a healthy diet including lots of fruits, vegetables is a key component of healthy lifestyles and plays a crucial role in maintaining a properly functioning and efficient immune system to defend against infections and other diseases. All the data was assessed from online search (PubMed, Google Scholar). In this review article we concentrate on vitamins and minerals.
Corresponding Author: Lata Kanyal Butola, Department of Biochemistry, Jawaharlal Nehru Medical College, Datta Meghe Institute of Medical Sciences, Sawangi, Wardha, Maharashtra, India. E-mail: kanyallata1010@gmail.com

\section{DOI: $10.14260 / j e m d s / 2020 / 497$}

How to Cite This Article:

Dhok A, Butola LK, Anjankar A, et al. Role of vitamins and minerals to improve immunity during covid-19 pandemic- a review. J. Evolution Med. Dent. Sci. 2020;9(32):22962300, DOI: 10.14260/jemds/2020/497

Submission 24-04-2020,

Peer Review 30-06-2020,

Acceptance 07-07-2020,

Published 10-08-2020.

Copyright (C) 2020 JEMDS. This is an open access article distributed under Creative Commons Attribution License [Attribution 4.0 International (CC BY 4.0)]

\section{KEY WORDS}

Immunity, Vitamins, Minerals, COVID-19 


\section{BACKGROUND}

The immune system has unique protective functions against infections. Bacteria that invade give raise to two radically different types of responses. Innate immunity is natural; its responses are similar to the infectious agent encountered, while acquired immunity is adaptive immunity; its responses rises strongly to repeated exposure to specific infection. Phagocytic cells, cells releasing inflammatory mediators and natural killers are types of innate responses.[1] Immunity is a diverse and redundant system that needs all of the nutrients to work properly. The immune response can be divided into three phases: reaction, control, and end reaction. The nutrient needs are greater during the response due to the need for mediator cell proliferation and synthesis.[2]

\section{Role of Vitamin C in Diet and Immunity}

Ascorbic acid was initially recognized as the scurvy disease prevention factor, also known as vitamin $\mathrm{C}$, and became very popular due to its antioxidant properties. This is a significant co-substrate of a wide class of enzymes, and it controls gene expression by interacting with specific transcription factors. Vitamin $\mathrm{C}$ is essential for all stressful conditions requiring immunity and being associated with inflammatory processes. For decades the accumulation of an inflammatory stimulus has been understood to be responsible for the onset of many diseases. Vitamin C plays an important role in eliminating potentially hazardous free radicals from the biological system, vitamin $\mathrm{C}$ helps to prevent heart disease, cancer, and also tends to slow the aging process. Vitamin $\mathrm{C}$ is important to stimulate the immune system by increasing the strength and defense of the organism. Consequently, their immune stimulant, antiinflammatory, antiviral and antibacterial roles are well established, and we have summarized their key functions in various forms of immune-related diseases and chronic inflammation. We may refer that vitamin $\mathrm{C}$ is suitable for use in different medicinal fields, including immunology, toxicology, radiobiology, and others because of its effects and variety of regulated pathways.[3]

\section{Role of Vitamin E in Diet and Immunity}

Strong antioxidant vitamin-E can modulate immune function of the host. It also has vital role in proper functioning of immune system, particularly in elderly population. Deficiency of vit-E has showed rise in cases of tumour and infections in host. On the other side, supplementation of vit-E has beneficial effect on immune system. High supplementation of vitamin $\mathrm{E}$ has shown beneficial effect to reduce aging, immunity of cell and degenerative diseases. ${ }^{[4]}$

\section{Role of Vitamin A in Diet and Immunity}

Vitamin A plays a varied part within the body. Vision, normal growth, fertility, fetal development, erythropoiesis, immune function, and antioxidant function are important. Liver, green leafy vegetables such as spinach and Amaranthus available in all seasons, whole milk, eggs, butter, cheese, fish, and meat are good sources of vit A. Deficiency of vit A affects immune systems and functioning at cellular level. There're numerous of study which has observed reduced or loss of phagocytic activity and impairment in functioning of B \& $\mathrm{T}$ cells in host with deficiency of vit-A. Its deficiency has also been reported to decrease natural killer activity, lower interferon production, decrease fix fat macrophage activity and response of lymphocytes in stimulation of mitogens. In the host with efficiency of vitamin A proper immune function and resistance to infection is observed.[5]

\section{Role of Vitamin D in Diet and Immunity}

Vitamin D is a modified steroid, synthesised in the skin under the influence of sunlight and is necessary for metabolism of calcium and phosphorus. Its RDA is $400 \mathrm{IU}$ or $10 \mathrm{mg}$, it binds to the receptor of target cells and regulate through gene expression. Vitamin D is present in good quantities in liver, butter cheese but on other side it has negative effect on $\mathrm{T}$ cell proliferation and Th1 type cytokine development ${ }^{[6]}$ also B-cell antibodies.[7] Th2 -type responses and its effects are not clear. ${ }^{[8]}$ and the number of regulatory $\mathrm{T}$-cells can increase. Overall, the existing evidence indicates that vitamin D is regulator of immune system and its effects depends on an immune function regulator and its effects on the immune situation (e.g. fitness, infectious disease, and autoimmune disorder).[9]

\section{Role of Iron in Immune System}

Iron is present in every cell of the body. Jaggery, organ meat, vegetables Leafy, pulses, cereals, bananas, dry fruits, and fish. There are a variety of studies of Fe's immune activity and the host vulnerability to infection, deficiency of iron causes thymus atrophy, which has many effects on the human immune system.[10] The main role of iron in humans is supplying oxygen to tissues and Co2 to the lungs as part of the haeme protein that is part of haemoglobin. Iron plays a key role in the transportation of the mitochondrial electron as a part of cytochromes and iron-sulfur proteins. Mainly it effects in broad spectrum and leads to impairment of the respiratory burst and killing of bacteria, proliferation of T-cells, and development of cytokines.[11] But the connection between deficiency of iron and vulnerability to infection remains undetermined. As a matter of fact, there is evidence that spending part of their life cycle infections triggered by microbes intracellularly, including plasmodia and mycobacteria, may theoretically be strengthened by Fe. Fe has been related to risk of malaria and infections in tropical infants at doses above an acceptable threshold, including pneumonia.[12] There are various reasons for the adverse effects of administering iron on infections. Whereas, Fe overload induces immune function impairment.[13]

\section{Role of Zinc in Immune System}

The human body has around $2 \mathrm{mg}$ of zinc in it. Meat, fish, nuts, legumes, eggs are good sources of zinc while zinc is not completely absorbed in cereals. Zinc plays a crucial role in the synthesis and stabilisation of DNA, RNA and Proteins, for the growth and differentiation of cells and for the protection of antioxidants, all of which are required for the function of immune cells. ${ }^{[14]}$ Zinc deficiency has a pronounced effect on the bone marrow which reduces the precursors of immune cells. ${ }^{[15]}$ It is an essential component of other enzymes in the 
body, such as carbonic anhydrase, alkaline phosphatase, alcohol dehydrogenase. It is essential for antioxidant function, as a component of superoxide dismutase. Zinc is important for the development of cytokine in monocytes and t-cells. So it is important for optimal function of immune system. Zinc is based on the accumulation and secretion of insulin from the pancreatic beta cells. Deficiency of zinc affects innate immunity including phagocytosis, the development of natural cell killers, and respiratory bursts.[16] There is significant effects of Zn deficiency on acquired immunity also, which leads to the decreases in the number of T-cells circulating and their function. ${ }^{[17]}$

\section{Role of Selenium in Diet and Immune System}

Seafood, muscle meat, and cereals of all kinds are rich sources of selenium, it is a component of a selenocysteine amino acid. Selenocysteine is incorporated into a very limited number of proteins directly during protein biosynthesis and it mainly occurs in the enzymes, namely glutathione peroxidase, deiodinase and thioreductase. Selenium is very much essential for the effective and efficient functioning of the immune system in both humans and animals, immune system protects species from pathogen attacks, immune functions also includes the inflammatory pathways if they are unregulated, leads to pathogenic conditions such as 'coronary heart disease, cancer, immunity, and rheumatoid arthritis'.[18,19] Selenium exercises synergetic antioxidant activity along with vitamin $\mathrm{E}$. In other words, selenium is complementary to vitamin E. It protects the membrane lipids, proteins, and other essential intracellular biomolecules from reactive oxygen attacks. Selenium is a part of glutathione peroxidase; it protects the organism from oxidant stress by catalysing the degradation of $\mathrm{H}_{2} \mathrm{O}_{2}$, hydroperoxides phospholipids, and other free radicals. It has been proposed that selenium's antioxidant effects were mediated by the glutathione peroxidases, the enzyme protects the organism against oxidant stress by catalysing the degradation of hydrogen peroxide and other free radicals. [20,21] Thus, selenium may work as an antioxidant in the extracellular space, along with cell membranes and particularly in the gastrointestinal tract, all with the potential to influence immune processes. Selenium is constituent of iodothyronine deiodinase, selenium is involved in removal of iodine from thyroid hormones. This process converts thyroxine to triiodothyroxine, it is also constituent of thioredoxin reductases an enzyme converting ribonucleotides to deoxyribonucleotide. Thioredoxin reductase is involved in immunological function and it also binds with heavy metals such as mercury and cadmium and protects the body from their toxic effects.[22]

\section{Role of Fruits and Vegetables}

Fruit and vegetables are shown in the dietary guidelines because of their high levels of 'dietary fiber, vitamins, minerals, especially electrolytes; and more recently, phytochemicals, especially antioxidants.'[23] Vegetable makes food palatable and bring variety to the diet. A daily intake of 60-80 $\mathrm{g}$ is recommended. Leafy vegetables are high in water content and dietary fibre. Roots and tubers include potato, carrots, colocasia, onion, yam and so on. They vary widely in the composition of nutrients. Carrots are good sources of beta carotene which is a natural antioxidant. The nutrients in fruits are readily available as they can be eaten raw and fresh. Fruits are good sources of minerals especially sodium and potassium. Custard apple (sitaphal) is rich in calcium. Dried fruits are good sources of calcium and iron. Papaya and mango have a high content of carotene. Guava, orange, amla are excellent sources of vitamin C. Banana and mango have high sugar content, hence good sources of energy. Optimal intake of fruit and vegetables was inked to a reduced risk of many noncommunicable diseases. Antioxidants that give bright colour to Fruits and vegetables and act as scavengers by eliminating free radicals before they have harmful health effects on the body.[24] In addition, dietary fibers found in Fruit and vegetables increases intestinal passage by forming a mass, resulting in more gradual nutrient absorption, ${ }^{[25]}$ and thus prevent constipation. Dietary fibre promotes the normal motility and produce a laxative effect. High fibre content improves glycaemic control and glucose tolerance and increase sensitivity to insulin. This benefits diabetic patients who can reduce their drug dose because of improved glucose tolerance. An increase in dietary fibre content reduces the incidence of colon cancers. They can be fermented in the colon, elevating the concentration of short-chain anticarcinogenic fatty acids,[26] and maintaining the gut health. Fibre reduces plasma lipid levels. It decreases the absorption of dietary cholesterol in the intestine. Fibre binds to bile salts and reduces their movements through enterohepatic circulation. These two mechanisms increase the excretion of cholesterol in the form of bile salts, by its hypolipidemic effect, it helps to prevent heart disease. Several studies have demonstrated Fruit and vegetables risk-reducing capacity for CVD, thereby preventing premature cardiovascular disorders.[27]

\section{Role of Vitamin B6 (Pyridoxine)}

Pyridoxine is an essential micronutrient for health and its deficiency could weaken the immunity,[28-30] including a decrease in serum antibody production and IL-2 production and an increase in IL-4. Plants contain B6 as pyridoxine while animal tissues contain PLP and phosphate pyridoxamine. Wheat bran, rice bran, dried yeast, legumes, nuts, meat, fish, milk, eggs, and leafy vegetables are rich sources of B6. Requirements contribute to protein consumption, as it is primarily concerned with the metabolism of amino acids. RDA is $2 \mathrm{mg} /$ day for adults. As normal it is slightly higher during pregnancy and lactation. Vitamin B6 mediates various in vivo metabolic processes mainly metabolism of amino acids, gluconeogenesis, lipid metabolism, and the development and functioning of nervous systems. Vitamin B6 was involved in controlling immune responses linked to a wide range of diseases and inflammation[31] and various cancers.[32-34] Previously many researches indicates that a deficiency in vitamin $\mathrm{B} 6$ leads to impairment of the immune responses.

\section{Role of Vitamin B12}

Normal daily requirement is about $1 \mu \mathrm{g}$. Vitamin $\mathrm{B}_{12}$ is also synthesised by bacteria in colon. It is not found in foods of plant origin. Hence, strict vegans who do not consume milk products are at risk of developing $\mathrm{B}_{12}$ deficiency. Liver, meat, fish, eggs, milk, curd and cheese are good sources of vitamin $\mathrm{B}_{12}$. In DNA synthesis the most important role of vitamin B 12 
is where cell replication is required. Vitamin B 12 acts in this cycle on folic acid. It is assumed that vitamin B 12, which plays a role in cell division, also serves as a modulator of human immunity; it stimulates the development of cellular immune recruited $\mathrm{T}$ lymphocytes, restores an abnormally increased CD4/ CD8 ratio, and retains normal lymphocyte subgroup counts.[35]

\section{CONCLUSIONS}

Immune system is highly dependent on micronutrients. Its deficiencies have high impact on the immune system. Proper amount of antioxidant neutralizer is useful in prevention of immune cells because phagocytes contain ROS as part of defence system against infection. Diet is considered as a good source for providing most of the antioxidants although a higher dose of antioxidants has been suggested to boost the body's immune system. The immune system operates at all times but in the presence of pathogens, unique immunity becomes more and more active. Carbohydrates, proteins and fats produces energy, involving electron transport chain and various coenzymes that are derivatives of vitamins. $\mathrm{Fe}$ and $\mathrm{Cu}$ are present in ETC which are essential for proper functioning of the immune cells. Vitamin A, D, other nutrients, and their metabolites regulate gene expression of immune cells. It also plays vital role in maturation, differentiation and responsiveness of immune cells in the host. In the light of above review, role of nutrients in maintenance of immune system is clearly observed and present review findings suggest that sufficient and balanced supply of nutrients is necessary for proper functioning of the immune system.

Financial or Other Competing Interests: None.

\section{REFERENCES}

[1] Delves PJ, Roitt IM. The immune system. First of two parts. N Engl J Med 2000;343(1):37-49.

[2] Percival SS. Nutrition and immunity: balancing diet and immune function. Nutrition Today 2011;46(1):12-7.

[3] Sorice A, Guerriero E, Capone F, et al. Ascorbic acid: its role in immune system and chronic inflammation diseases. Mini Rev Med Chem 2014;14(5):444-52.

[4] Moriguchi S, Muraga E. Vitamin E and immunity. Vitam Horm 2000;59:305-36.

[5] Meydani SN, Fawzi WW, Sun NH. The effect of vitamin deficiencies ( $E$ and $A$ ) and supplementation on infection and immune response. In: Tontisirin K, Suskind R, ed. Nutrition, immunity and infection disease in infants and children, 45th Nestle Nutrition Workshop. Nestle Nutrition Services 2001;45:213-41.

[6] Lemire JM, Archer DC, Beck L, et al. Immunosuppressive actions of 1,25-dihydroxyvitamin D3: preferential inhibition of Th1 functions. J Nutr 1995;125(6 Suppl):1704S-8.

[7] Lemire JM, Adams JS, Sakai R, et al. 1 alpha,25dihydroxyvitamin D3 suppresses proliferation and immunoglobulin production by normal human peripheral blood mononuclear cells. J Clin Invest 1984;74(2):657-61.
[8] Boonstra A, Barrat FJ, Crain C, et al. 1alpha,25dihydroxyvitamin $\mathrm{d} 3$ has a direct effect on naive $\mathrm{CD} 4+\mathrm{T}$ cells to enhance the development of Th2 cells. J Immunol 2001;167(9):4974-80.

[9] Gregori S, Giarratana N, Smiroldo S, et al. A 1alpha,25dihydroxyvitamin $\mathrm{D}(3)$ analog enhances regulatory $\mathrm{T}$ cells and arrests autoimmune diabetes in NOD mice. Diabetes 2002;51(5):1367-74.

[10] Sherman AR, Spear AT. Iron and immunity. In: Klurfeld DM, ed. Nutrition and immunology. New York and London: Plenum Press 1993:285-307.

[11] Kumar V, Choudhry VP. Iron deficiency and infection. Indian J Pediatr 2010;77(7):789-93.

[12] Murray MJ, Murray AB, Murray MB, et al. The adverse effect of iron repletion on the course of certain infections. Br Med J 1978;2(6145):1113-5.

[13] Oppenheimer SJ. Iron and its relation to immunity and infectious disease. J Nutr 2001;131(2S-2):616S-35S.

[14] Iwata M, Hirakiyama A, Eshima Y, et al. Retinoic acid imprints gut-homing specificity on $\mathrm{T}$ cells. Immunity 2004;21(4):527-38.

[15] Fraker PJ, King LE, Garvy BA, et al. The immunopathology of zinc deficiency in humans and rodents: a possible role for programmed cell death. In: Klurfeld DM, ed. Nutrition and Immunology. New York and London: Plenum Press 1993:267-83.

[16] Fraker PJ, King LE. Reprogramming of the immune system during zinc deficiency. Annu Rev Nutr 2004;24:277-98.

[17] Allen JL, Perri RT, McClain CJ, et al. Alterations in human natural killer cell activity and monocyte cytotoxicity induced by zinc deficiency. J Lab Clin Med 1983;102(4):577-89.

[18] Kryukov GV, Gladyshev VN. Mammalian selenoprotein gene signature: identification and functional analysis of selenoprotein genes using bioinformatic methods. In: Sies H, Packer L, eds. Methods in enzymology 347. San Diego, CA: Academic Press 2002:84-100.

[19] Lescure A, Gautheret D, Krol A. Novel selenoproteins identified from genomic sequence data. In: Sies H, Packer L, eds. Methods in enzymology 347. San Diego, CA: Academic Press 2002:57-70.

[20] Arthur JR. The glutathione peroxidases. Cell Mol Life Sci 2000;57(13-14):1825-35.

[21] Pfeifer H, Conrad M, Roethlein D, et al. Identification of a specific sperm nuclei selenoenzyme necessary for protamine thiol cross-linking during sperm maturation. FASEB J 2001;15(7):1236-8.

[22] Miller S, Walker SW, Arthur JR, et al. Selenite protects human endothelial cells from oxidative damage and induces thioredoxin reductase. Clin Sci (Lond) 2001;100(5):543-50.

[23] Slavin JL, Lloyd B. Health Benefits of fruits and vegetables. Adv Nutr 2012;3(4):506-16.

[24] Kaur C, Kapoor HC. Antioxidants in fruits and vegetables: the millennium's health. Int J Food Sci Technol 2001;36(7):703-25.

[25] Anderson JPS, Young L, Prior S. Dietary fibre fact. Sheet No. 9.333. Colorado State University: Fort Collins, CO, USA 2010. http://www.ext.colostate.edu/pubs/foodnut/ 09333.pdf 
[26] Lattimer JM, Haub MD. Effects of dietary fibre and its components on metabolic health. Nutrients 2010;2(12):1266-89.

[27] Adebawo O, Salau B, Ezima E, et al. Fruits and vegetables moderate lipid cardiovascular risk factor in hypertensive patients. Lipids Health Dis 2006;5:14.

[28] Kumar M, Axelrod AE. Cellular antibody synthesis in vitamin B6-deficient rats. The Journal of Nutrition 1968;96(1):53-9.

[29] Doke S, Inagaki N, Hayakawa T, et al. Effect of vitamin B6 deficiency on an antibody production in mice. Biosci Biotechnol Biochem 1997;61(8):1331-6.

[30] Inubushi T, Okada M, Matsui A, et al. Effect of dietary vitamin $\mathrm{B} 6$ contents on antibody production. BioFactors 2000;11(1-2):93-6.

[31] Lotto V, Choi SW, Friso S. Vitamin B6: a challenging link between nutrition and inflammation in CVD. Br J Nutr 2011;106(2):183-95.
[32] Rimando AM, Suh N. Natural products and dietary prevention of cancer. Mol Nutr Food Res 2008;52 Suppl 1:S5.

[33] Wu XY, Lu L. Vitamin B6 deficiency, genome instability and cancer. Asian Pac J Cancer Prev 2012;13(11)5333-8.

[34] Galluzzi L, Vacchelli E, Michels J, et al. Effects of vitamin B6 metabolism on oncogenesis, tumor progression and therapeutic responses. Oncogene 2013;32(42):49955004.

[35] Tamura J, Kubota K, Murakami $H$, et al. Immunomodulation by vitamin B 12: augmentation of CD8+ T lymphocytes and natural killer (NK) cell activity in vitamin B 12 -deficient patients by methyl-B 12 treatment. Clin Exp Immunol 1999;116:28-34. 